\title{
Design, Simulation and Comparison of Different Stage Amplifiers Using 2N2222A Transistor
}

\author{
Y. Abdullahi ${ }^{1 *}$ and Mu'azu Musa ${ }^{2}$ \\ ${ }^{1}$ Department of Science Laboratory Technology, Umaru Ali Shinkafi Polytechnic Sokoto, Nigeria \\ ${ }^{2}$ Department of Mechanical Engineering, Usmanu Danfodiyo University, Sokoto
}

Nigeria

\begin{abstract}
The output from a single-stage amplifier is usually insufficient to drive an output device. In other words, the gain of a single amplifier is inadequate for practical purposes. Consequently, additional amplification over two or three stages is necessary. To achieve this, the output of each amplifier stage is coupled in some way to the input of the next stage. The resulting system is referred to as multistage amplifier. In this paper, a complete common of $2 \mathrm{~N} 2222 \mathrm{~A}$ transistor amplifier using RC coupled for single-stage and multistage (two stages, three stages), was designed. to analyze bandwidth using MULTISIM.11 software. The gain of the RC coupled was simulated and the Gain plot is used for the given amplifier to know the lower cut off frequency (Hz), upper cut off frequency $(\mathrm{Hz})$ and bandwidth $(\mathrm{BW})$, which gives the range of the signals that the amplifier can process. The result shows that multi-stage amplifiers are best suited for the applications which require a high input resistance, low output resistance, and large gains.
\end{abstract}

Key Words: Amplifiers, Simulation, 2N2222A, Transistor, Multistage.

\subsection{INTRODUCTION}

In practice amplifier which can amplify a signal from a very weak source such as a microphone, to a level which is suitable for the operation of another transducer such as loudspeaker This is achieved by cascading number of amplifier stages, known as multistage amplifier [1]. In cascade arrangement, output of first stage is connected to the input of the second stage through a suitable coupling device. The purpose of coupling device (e.g. a capacitor, transformer etc.) is to transfer a.c. output of one stage to the input of the next stage; and to isolate the d.c. conditions of one stage from the next stage [2]. Figure 1.0 shows the block diagram of a 3-Stage Amplifier. Each stage consists of one transistor and associated circuitry iscoupled to the next stage through a coupling device. The name of the amplifier is usually given after the type of coupling used [1].

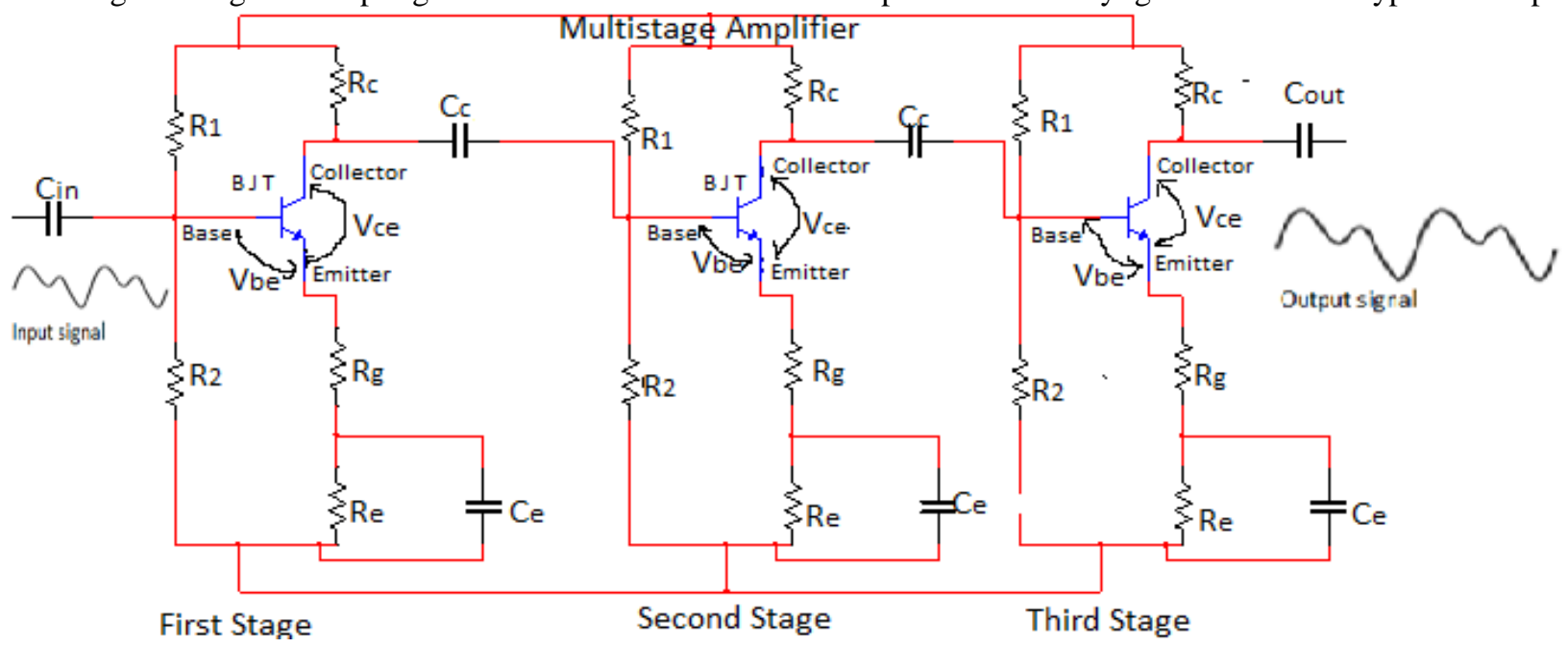

Figure 1: Common Emitter 3-Stage Amplifier Transistor Amplifier Circuit 
The coupling method can be $: R C$ coupling, Transformer coupling or Direct coupling in which the multistage amplifier is referred to as: R-C coupled amplifier, Transformer coupled amplifier and Direct coupled amplifier [3].

The theory of transistor reveals that it will function properly if its input circuit (i.e. base-emitter junction) remains forward biased and output circuit (i.e. collector-base junction) remains reverse biased at all times [2]. This is then the key factor for achieving faithful amplification.

The process of raising the strength of a weak signal without any change in its general shape is known as Faithful Amplification

[4]. To ensure this, the following basic conditions (known as transistor biasing) must be satisfied [5]:

(i) Proper zero signal collector current;

(ii) Minimum proper base-emitter voltage $\left(V_{b e}\right)$ at any instant; and

(iii) Minimum proper collector-emitter voltage $\left(V_{c e}\right)$ at any instant.

Essentials of a Transistor Biasing Circuit are [2]:

a) It should ensure proper zero signal collectors current;

b) It should ensure that $\mathrm{V}_{\text {ce }}$ does not fall below $0.5 \mathrm{~V}$ for Ge transistors and $1 \mathrm{~V}$ for silicon transistors at any instant;

c) It should ensure the stabilization of operating point which can be achieved with a bias battery.

The most commonly used methods of obtaining transistor biasing from one source of supply (i.e. $\mathrm{V}_{\mathrm{cc}}$ ) are [6]:
A. Base resistor method;
B. Emitter bias method;
C. Biasing with collector-feedback resistor;
D. Voltage-divider bias.

The most widely used method of providing biasing and stabilization to a transistor is Voltage-divider bias. In voltage divider method, two resistances $R_{1}$ and $R_{2}$ are connected across the supply voltage $V_{c c}$ and provide biasing as shown in Figure 2 . The emitter resistance $R_{e}$ provides stabilization. The name "voltage divider" comes from the voltage divider formed by $R_{1}$ and $R_{2}$. The voltage drop across $R$ forward biases the base-emitter junction. This causes the base current and hence collector current flows in the zero signal conditions.

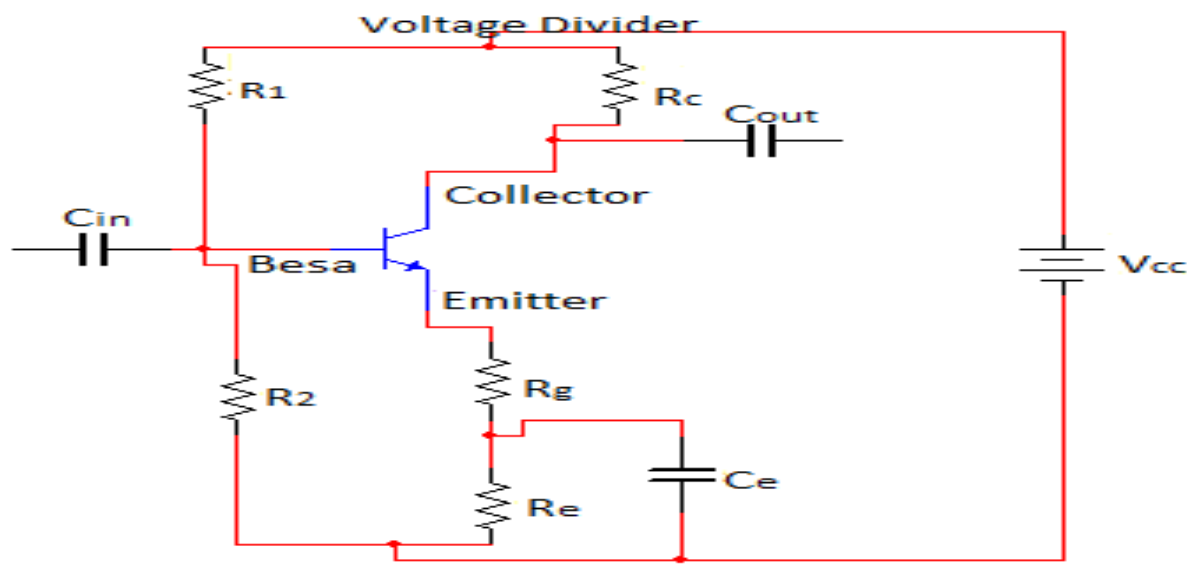

Figure 2: Common Emitter Voltage Divider Transistor Amplifier Circuit

\subsection{Transistor Amplifier Design}

To design a transistor circuit, the values of the following components are to be determined [7]:

i. Battery Voltage, $V_{c c}$;

ii. Operating Point;

iii. Collector and Emitter Resistors;

iv. Choice of the Bias Resistors $\mathrm{R}_{1}$ and $\mathrm{R}_{2}$;

v. Choice of $\mathrm{Rg}$;

vi. Choice of Capacitors

vii. Complete Circuit Diagram.

\subsection{Choosing Battery Voltage $\left(V_{c c}\right)$}

The battery voltage, $V_{c c}$ must be less than the maximum voltage the transistor can handle between the collector and emitter [8] . From the data sheet absolute maximum rating, $\mathrm{V}_{\mathrm{CEO}}$ of $2 \mathrm{~N} 2222 \mathrm{~A}$ Transistor is $40 \mathrm{~V}$ [9]. Based on suitability and availability, $V_{c c}$ is chosen to be $12 \mathrm{~V}$. 
International Journal of Advances in Scientific Research and Engineering (ijasre),Vol 5 (9), September-2019

\subsection{Choosing an Operating Point}

Operating Point is the DC values of $I_{c}, I_{b}$, and $V_{c e}$ which are the steady state values [10].The point is to be chosen as follows:

i. $\quad$ Choose an $\mathrm{I}_{\mathrm{C}}$ such that the transistor actually does amplify;

ii. Given the value of $\mathrm{I}_{\mathrm{C}}$, and gain, the base current $\mathrm{I}_{\mathrm{B}}$ at the operating point is determined from:

$$
I_{b}=\frac{I_{C}}{\beta}
$$

iii. The operating point collector-emitter voltage, $V_{c e}$ is determined within the range as:

$$
\frac{V_{\text {battery }}}{3}<V_{c e}<\frac{V_{\text {battery }}}{2}
$$

A $V_{c e}$ somewhere in this range will allow for amplification of a maximum input voltage without distortion. For this design, $V_{\text {battery }}=12 \mathrm{~V} ; \beta=200$. Substituting these values gives in to equation 2 we have

$$
V_{c e}=\frac{V_{\text {battery }}}{3}=\frac{12}{3}=4 \mathrm{~V}
$$

For this value, the transistor might have

$$
I_{c}=4 \mathrm{~mA} \text {. }
$$

Therefore, $I_{b}=\frac{4 \times 10^{-3}}{200}=0.00002=0.02 \mathrm{~mA}=20 \mu \mathrm{A}$

This value might be just large enough for measuring.

These value of $V_{c e}=4 \mathrm{~V}, I_{c}=4 \mathrm{~mA}$ and $I_{b}=0.02 \mathrm{~mA}$ when $\beta=200$, is known as DC operating point.

\subsection{Choosing the Collector and Emitter Resistors}

According to the rule: $R_{e} \approx R_{c}$ and $I_{c} \approx I_{e}$ [10].

Also from Kirchoff's loop rule as cited in Johnson [11]:

$$
\begin{aligned}
& V_{c c}=R_{c} I_{c}+V_{c e}+I_{e} R_{e} \\
& R_{c}=\frac{V_{c c}-V_{c e}}{2 I_{c}} \\
& R_{c}=\frac{12-4}{2 \times 4 \times 10^{-3}}=\frac{8}{0.008}=1000 \Omega
\end{aligned}
$$

$\therefore R_{c}=R_{e}=1000 \Omega=1 \mathrm{k} \Omega$

2.4The Choice of the Bias Resistors $R_{1}$ and $R_{2}$

According to Johnson [11]:

$$
I_{2} R_{2}=V_{b e}+V_{e}
$$

But $V_{c c}=V_{c e}+V_{e}$ and $V_{e}=V_{c c}-V_{c e}$

Therefore: $V_{e}=12-4=8 V$ and $\quad V_{b e}=0.6 \mathrm{~V}$

This makes: $I_{2} R_{2}=0.6+8=8.6 \mathrm{~V}$ and $\quad R_{2}=\frac{8.6}{I_{2}}$

Also,

$$
V_{c c}-R_{1} I_{1}-V_{b e}=0
$$

But $I_{1}=\left(I_{2}+I_{b}\right)$

Therefore,

$$
R_{1}=\frac{V_{c c}-V_{b e}}{\left(I_{2}+I_{b}\right)}
$$

It is a good idea to choose $I_{2} \gg I_{b}$

$I_{2}=25 \times I_{b}=25 \times 0.00002=0.0005 \mathrm{~A}$. (Neglecting $\left.I_{b} \ll I_{2}\right)$

$R_{1}=\frac{12-0.6}{0.0005}=\frac{11.6}{0.0005}=22800 \Omega=22.8 \mathrm{k} \Omega$ and $R_{2}=\frac{8.6}{0.0005}=17200 \Omega=17.2 \mathrm{k} \Omega$

The values of $R_{1}=22.8 \mathrm{k} \Omega$ and $R_{2}=17.2 \mathrm{k} \Omega$ was chosen. 
International Journal of Advances in Scientific Research and Engineering (ijasre),Vol 5 (9), September-2019

\subsection{The Choice of $\mathbf{R}_{\mathrm{g}}$}

The entire AC input voltage appears across $R_{g}$ so that in terms of the AC emitter current $I_{e}$ we have from Ohm's law [12].

$$
i_{e}=\frac{V_{\text {input }}}{R_{g}}
$$

The output capacitor serves to block the DC from the output voltage. The output AC voltage $V_{\text {output }}$ is given by:

$$
V_{\text {output }}=i_{c} R_{c}=i_{e} R_{c}
$$

Since $i_{e} \approx i_{c}$, therefore:

$$
V_{\text {output }}=\frac{R_{C}}{R_{g}} V_{\text {input }}
$$

From AC gain, $\mathrm{g}$ is:

$g=\frac{V_{\text {output }}}{V_{\text {input }}}=\frac{R_{C}}{R_{g}}$

Therefore,

$$
R_{g}=\frac{R_{C}}{g}
$$

This is the chosen $\mathrm{Rg}$ for a particular gain.

\subsection{The Values of the Capacitors}

The resistor and capacitor act as a voltage divider of the input voltage. The voltage across the resistor $V_{R}$ is [12]:

$$
V_{R}=\frac{R}{\sqrt{R^{2}+\frac{1}{\omega^{2} c^{2}}}} V_{\text {input }}
$$

Where $\omega=2 \pi f$ and $\mathrm{f}$ is the frequency of the signal generator that provides the input voltage. The resistor $R=R_{2}$ and the capacitor $c=C_{\text {input }}$. If the amplifier is for audio frequencies, then the lowest $\mathrm{f}=20 \mathrm{~Hz}$.

Choose C so that $V_{R}=\frac{V_{\text {input }}}{2}$ at the lowest audio frequency so that at the lowest audio frequency, half the input voltage appears across the resistor. So the equation can be write as:

$$
\begin{aligned}
& v_{R}=\frac{V_{\text {input }}}{2}=\frac{2 V_{R}}{V_{\text {input }}}=\frac{V_{\text {input }}}{V_{\text {input }}}=1 \\
& \frac{V_{R}}{V_{\text {input }}}=\frac{1}{2} \\
& \frac{1}{2}=\frac{R}{\sqrt{R^{2}+\frac{1}{\omega^{2} c^{2}}}} \\
& 4 R^{2}=R^{2}+\frac{1}{\omega^{2} c^{2}} \\
& 4 R^{2} \omega^{2} c^{2}=\omega^{2} c^{2} R^{2}+1 \\
& 3 R^{2} \omega^{2} c^{2}=1 \\
& w^{2}=(2 \times 3.142 \times 20)^{2}=1.5795462 \times 10^{4} \mathrm{rad} / \mathrm{sec} \\
& \therefore C_{\text {input }}=\frac{1}{\sqrt{3 R_{2}^{2} \omega^{2}}}=\frac{1}{3.7441672 \times 10^{06}}=0.000000267=0.267 \times 10^{-6}=0.267 \mu F .
\end{aligned}
$$

This is the value of input capacitor. The output capacitor is determined by similar reasoning

The voltage across the resistor $V_{R}$ is

$$
\begin{aligned}
& V_{R}=\frac{R}{\sqrt{R^{2}+\frac{1}{\omega^{2} c^{2}}}} V_{\text {output }} \\
& \text { But } V_{R}=\frac{V_{\text {output }}}{2}
\end{aligned}
$$


International Journal of Advances in Scientific Research and Engineering (ijasre),Vol 5 (9), September-2019

$$
\frac{V_{R}}{V_{\text {output }}}=\frac{1}{2}
$$

$\therefore C=\frac{1}{\sqrt{3 R^{2} \omega^{2}}}$

Where, $R^{2}=R_{c}^{2}=(1000)^{2} \Omega, \omega^{2}=(2 \pi f)^{2}=(2 \times 3.142 \times 20)^{2}=1.5795462 \times 10^{2} \mathrm{rad} / \mathrm{sec}$, and $c=C_{\text {output }}$. Substituting this value we have

$C_{\text {output }}=4.5938118 \times 10^{-6}=4.5938118 \mu \mathrm{F}$. This is the output capacitor.

Choosing the emitter capacitor, the emitter capacitor can be a short circuit for $\mathrm{AC}$ around the emitter resistor. The time constant of the resistor and capacitor is $T=R C$ [13]. Where $T=\frac{1}{f}$ and $f=20 H_{Z}$ is the lowest AC frequency, $R=R_{e}$ is already known $(R=1000 \Omega)$. So the value of $C=C_{e}$ can be compute specifically as

From $T=R C$

$C=\frac{T}{R}$ where, $T=\frac{1}{f}$ and $f=20 H_{Z}, R=R_{e}=1000 \Omega, C=C_{e}$

$\therefore C_{e}=\frac{T}{R_{e}}$

$C_{e}=\frac{0.05}{100}=0.00005=50 \times 10^{-6}=50 \mu F$. This is value of emitter capacitor.

2.7 The Complete Common Emitter Transistor Amplifier

Base of the values of $R_{c}=R_{e}=1 \mathrm{~K}, R_{1}=22.8 \mathrm{~K} \Omega, R_{2}=17.2 \mathrm{~K} \Omega, C_{\text {input }}=0.267 \mu \mathrm{F}, C_{\text {output }}=4.593811 \mu \mathrm{F}, C_{e 3}=50 \mu \mathrm{F}$, $V_{c c}=12 \mathrm{~V}$ since $12 \mathrm{~V}$ is readily available in the lab and $R_{g}=\frac{R_{c}}{g}$ which is the chosen for a particular gain. The complete common emitter transistor amplifier circuit is indicated below [14]:

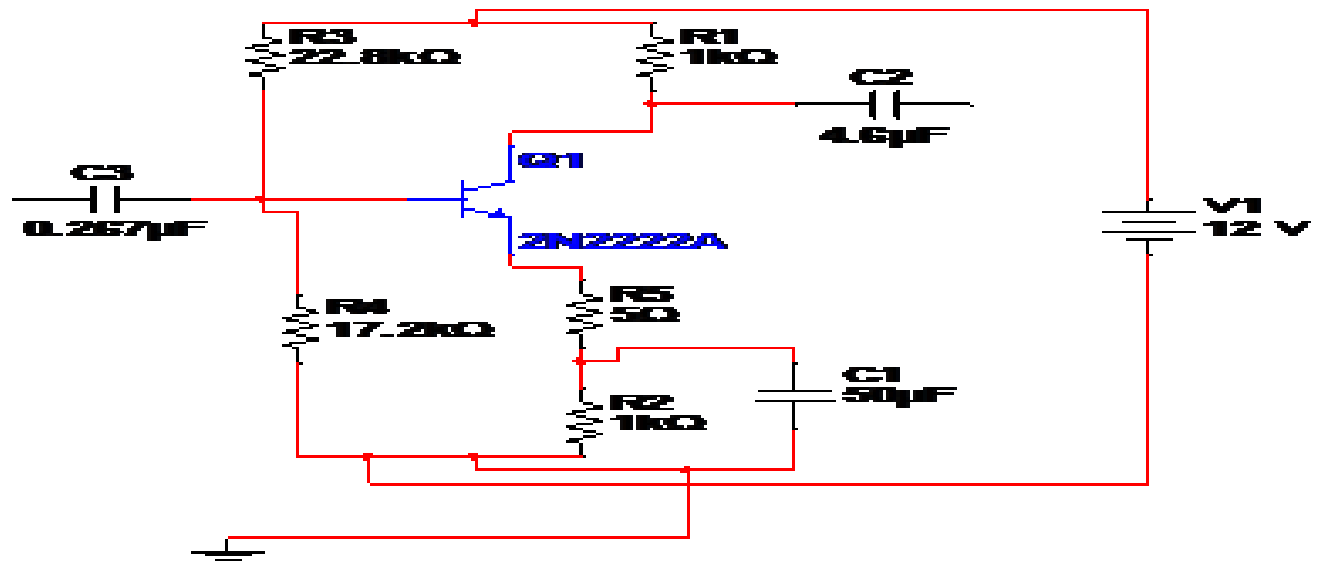

Figure 3. Single StageTransistor Amplifier Circuit

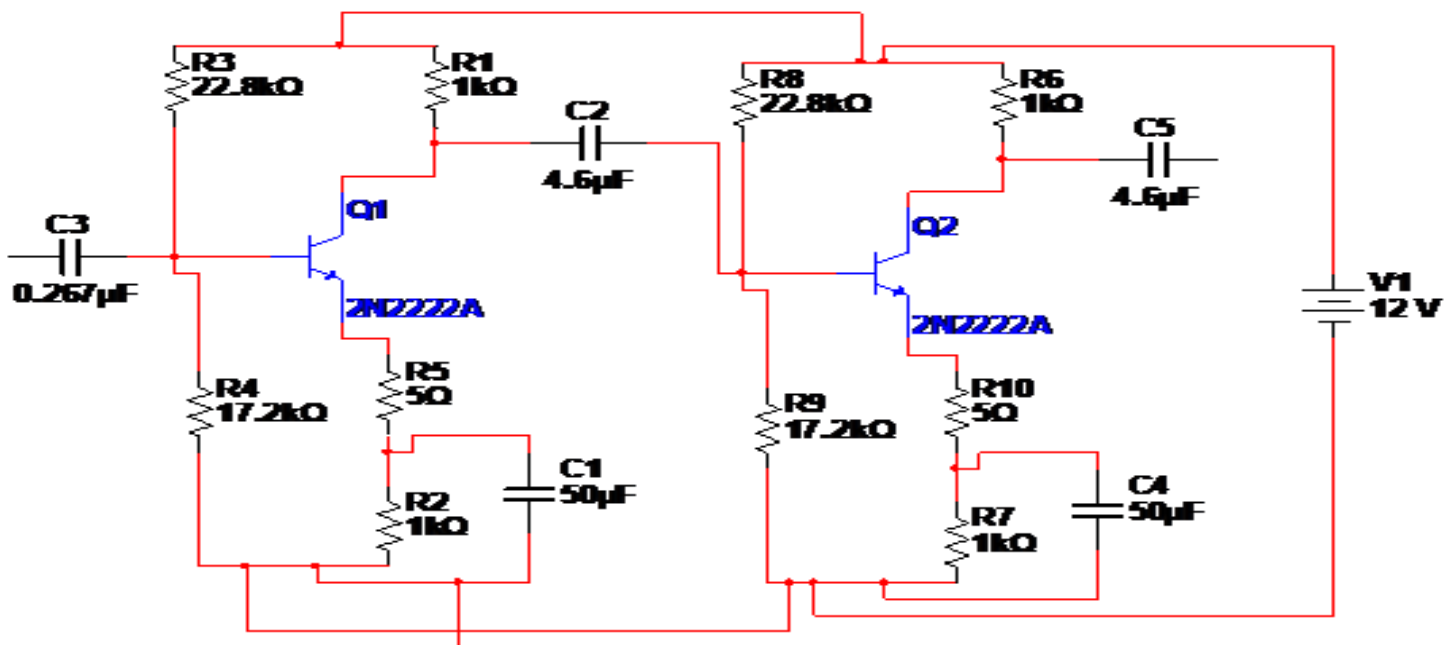

Figure 4. Double StageTransistor Amplifier Circuit 


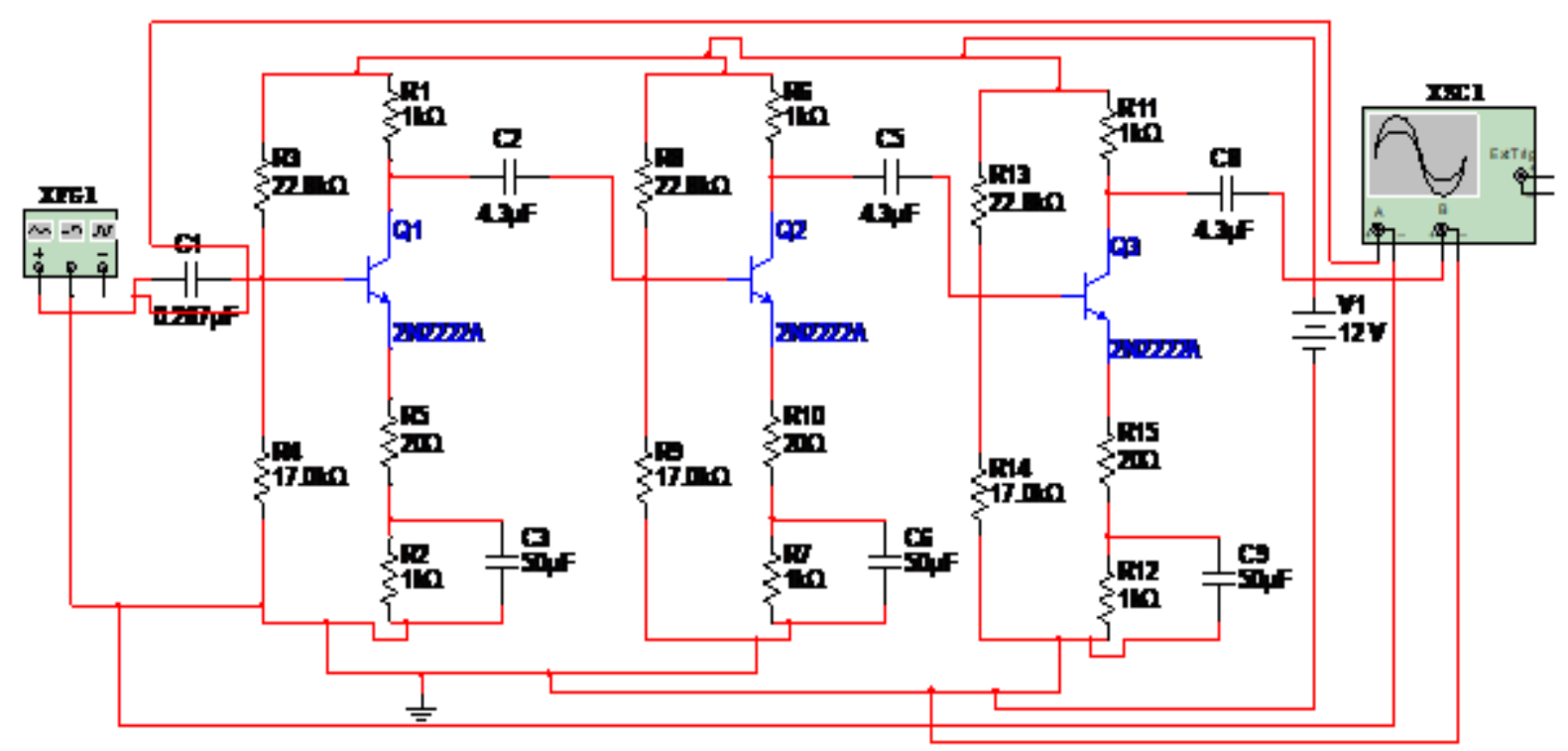

Figure 5. Multi StageTransistor Amplifier Circuit

\subsection{SIMULATED PERFORMANCE}

Circuit simulations were performed using the Multisim11.0.1 simulator. Function generator was used to provide the amplifier input signal for test. For all tests, the function generator Frequency was set to provide a peak-to-peak sinusoidal input to the amplifier. Based on selected values for the implementation, the corresponding simulated gains are also indicated in the table for more detailed plots of the simulated performance are given where the simulated curves using measured component values are presented.

\subsection{Results and Discussions}

Frequency response of an electric or electronics circuit allows us to see exactly how the output gain (known as the magnitude response) and the phase (known as the phase response) changes at a particular single frequency, or over a whole range of different frequencies from $\mathrm{OHz}$, to many thousands of mega-hertz, $(\mathrm{MHz})$ depending upon the design characteristics of the circuit.

For single stage, at constant Resistor $\operatorname{Rg}(5 \Omega)$ and constant Input Voltage $(10 \mathrm{mV})$ varying input Frequency, the Gain was determined graphically shown in below.

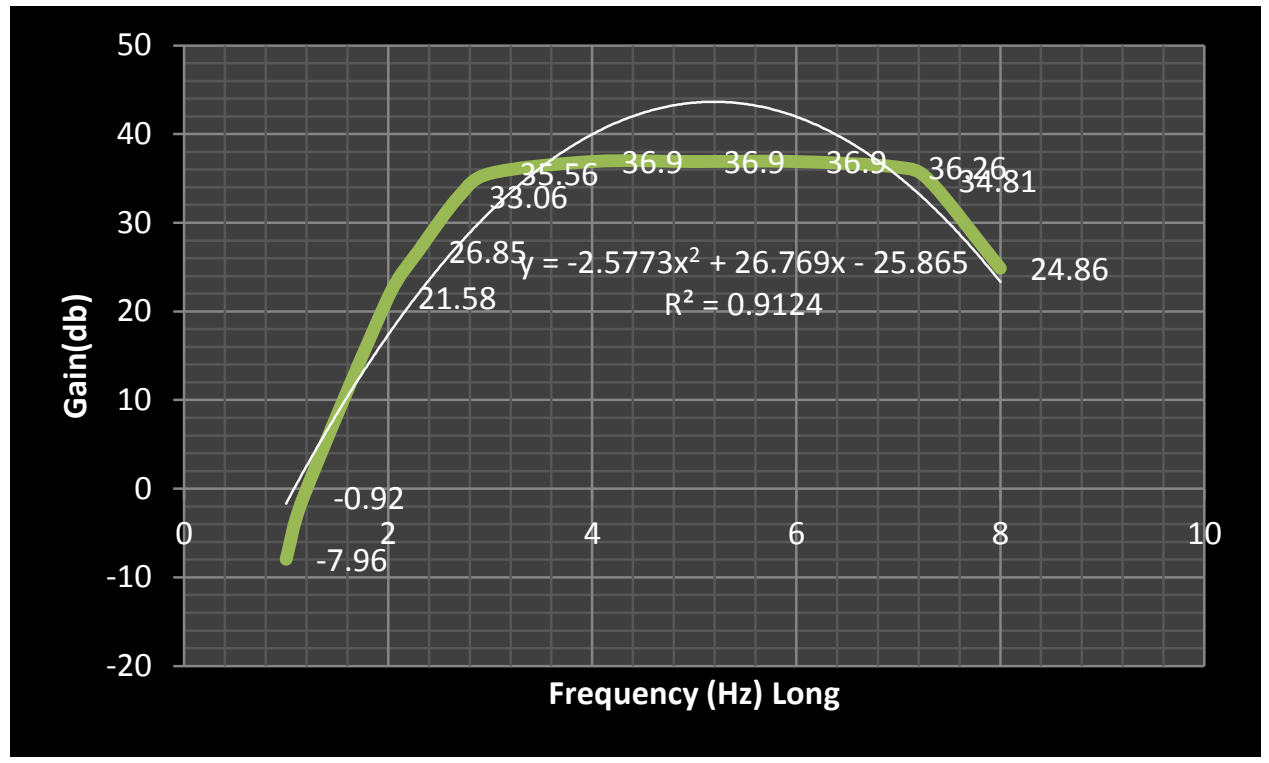

Figure 6: A typical Frequency- versus-Gain curve For Single Stage Amplifier

A typical Gain $(\mathrm{db})$ - versus- Frequency curve is shown in Figure 6. While analyzing this curve, three value of frequency are important mid-frequency range ,Log 3 - Log 7, lower cut-off frequency, Log35.56, upper cut-off frequency, $\log 36.26$.The cut-off 
frequencies are also called roll-off frequencies because at these frequencies the amplifier Gain starts rolling down from its midband value or maximum value. The frequency span between these two cut-off frequencies is called the passband or bandwidth (BW) of the amplifier. All frequencies lying between lower cut- off and upper cut - off are amplified almost equally. It indicates that the Frequency at which the amplifier instantaneously generates Gain can be observed by looking at the first data point that has Long 2, Gain(db) of 21.58 and Frequency $\log 2.30$, Gain(db) of 26.85 , these indicate that increases in Frequency would cause increases in Gain produced by the amplifier.It can be observed from graph that the empirical relation between Gain and Frequency is $\mathbf{y}=-\mathbf{2 . 5 7 7} \mathbf{x}^{\mathbf{2}}+\mathbf{2 6 . 7 6 x}-\mathbf{2 5 . 8 6}$ this is the polynomial relation which is the best among the different relation. The coefficient of determination $\mathbf{R}^{\mathbf{2}}=\mathbf{0 . 9 1 2}$ which indicate that the correlation is very high. The equation can be used to estimate the Gain at any particular Frequency.

For Double Stage, at constant Resistor $\operatorname{Rg}(5 \Omega)$ and constant Input Voltage $(1 \mathrm{mV})$ varying input Frequency, the Gain was determined graphically shown in below

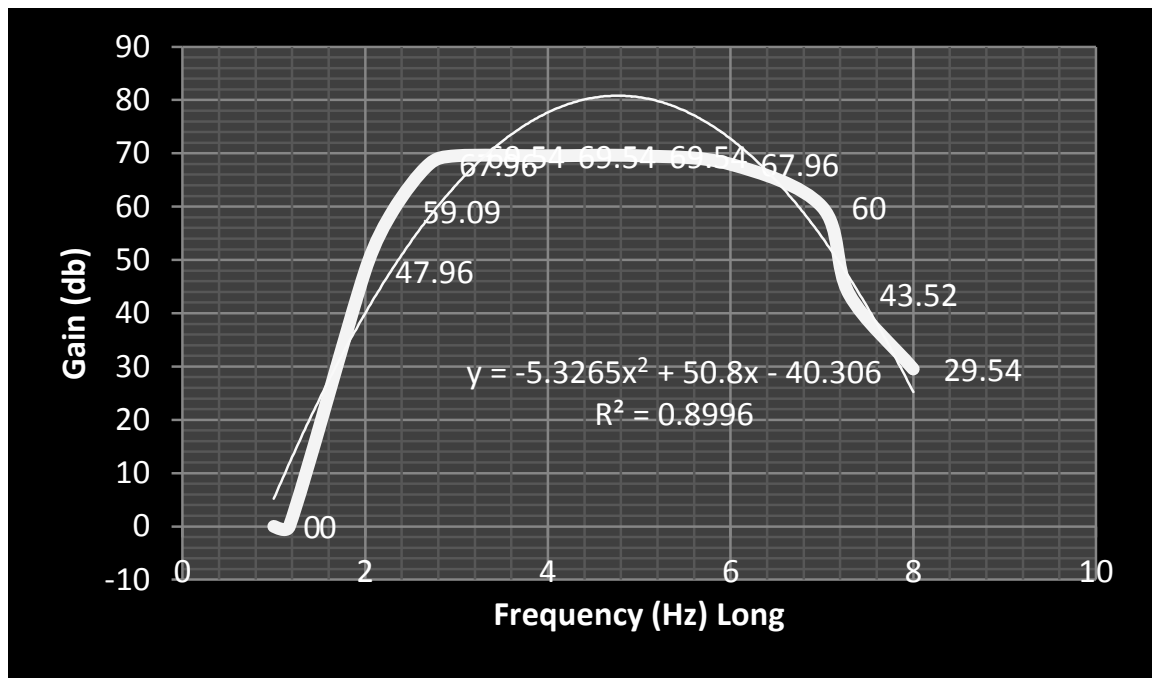

Figure 7: A typical Frequency- versus-Gain curve For Single Stage Amplifier

A typical Gain $(\mathrm{db})$ - versus- Frequency curve is shown in Figure 7 . While analyzing this curve, three value of frequency are, mid-frequency range , $\log 2.70$ - $\log 6$, lower cut-off frequency, $\log 59.09$, upper cut-off frequency, log 60.00 , all frequencies lying between lower cut- off and upper cut - off are amplified almost equally. It indicates that the Frequency at which the amplifier instantaneously generates Gain can be observed by looking at the first data point that has Long 2, Gain(db) of 47.96 and Frequency $\log 2.3$, Gain(db) of 59.09, these indicate that increases in Frequency would cause increases in Gain produced by the amplifier.It can be observed from graph that the empirical relation between Gain and Frequency is $\mathbf{y}=-\mathbf{5 3 2 6} \mathbf{x}^{\mathbf{2}}+\mathbf{5 0 . 8} \mathbf{x}-$ 40. 30this is the polynomial relation which is the best among the different relation. The coefficient of determination $\mathbf{R}^{\mathbf{2}}=\mathbf{0 . 8 9 9}$ which indicate that the correlation is very high. The equation can be used to estimate the Gain at any particular Frequency.

For Third Stage, at constant Resistor $\operatorname{Rg}(5 \Omega)$ and constant Input Voltage $(1 \mu \mathrm{V})$ varying input Frequency, the Gain was determined graphically shown in below

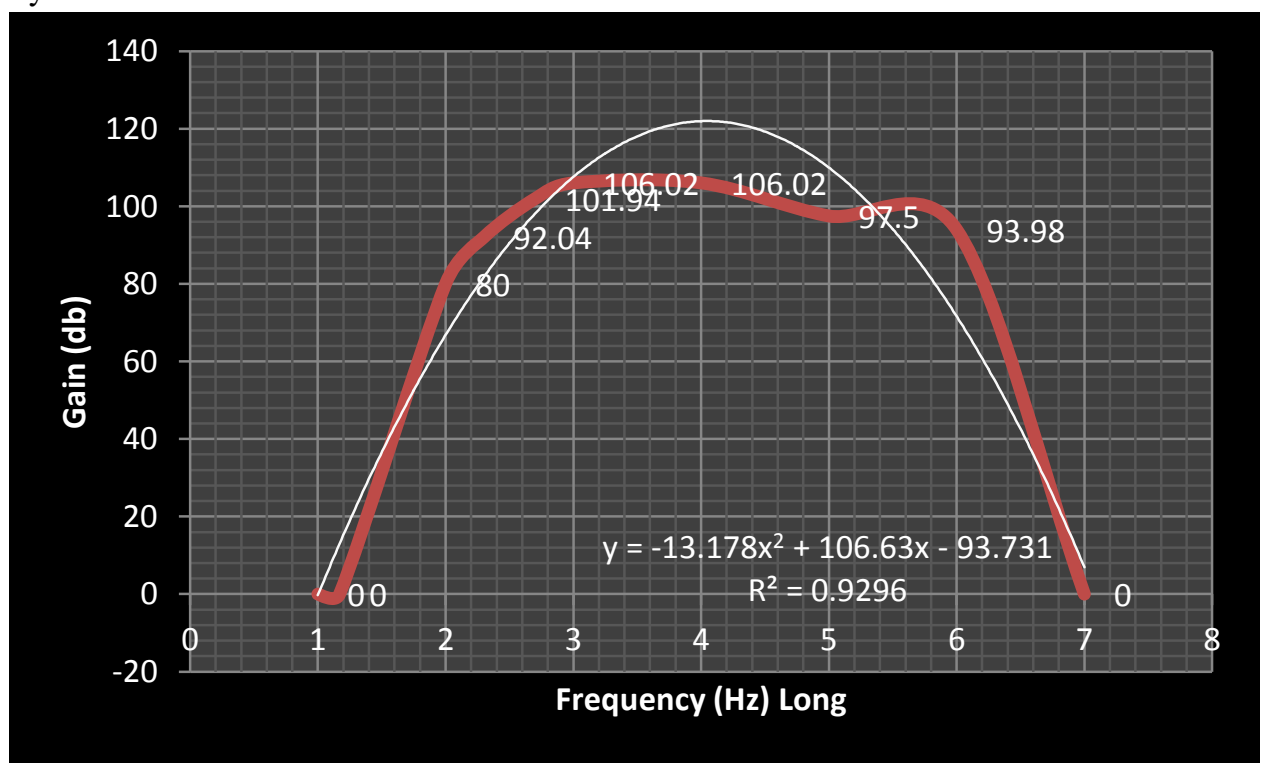

Figure 8: A typical Frequency- versus-Gain curve For Single Stage Amplifier 
A typical Gain $(\mathrm{db})$ - versus- Frequency curve is shown in Figure 8 While analyzing this curve, three value of frequency are, midfrequency range ,Log 2.70 - Log 5, lower cut-off frequency, $\log 92.04$, upper cut-off frequency, $\log 93.98$, all frequencies lying between lower cut- off and upper cut - off are amplified almost equally. It indicates that the Frequency at which the amplifier instantaneously generates Gain can be observed by looking at the first data point that has Long 2, Gain(db) of 80 and Frequency $\log 2.30$, Gain $(\mathrm{db})$ of 92.04 , these indicate that increases in Frequency would cause increases in Gain produced by the amplifier.It can be observed from graph that the empirical relation between Gain and Frequency is $\mathbf{y}=\mathbf{- 1 3 . 1 7} \mathbf{x}^{\mathbf{2}}+\mathbf{1 0 6 . 6} \mathbf{x}-$ 93. 73this is the polynomial relation which is the best among the different relation. The coefficient of determination $\mathbf{R}^{\mathbf{2}}=\mathbf{0 . 9 2 9}$ which indicate that the correlation is very high. The equation can be used to estimate the Gain at any particular Frequency.

\section{CONCLUSION}

The design and implementation of a 2N2222A Transistor Multistage Amplifiers has been presented. The amplifiers were simulated using Multisim 11.0.1 soft ware. The tests verified the performance of the amplifiers. It indicates the Frequency at which the amplifiers instantaneously generates Gain, the rang of the Input Frequency at which the amplifiers has different Gain, the rang it has constant Gain and rang at which the Gain was gradually decreasing with increasing Input Frequency which is called saturation point. The cut-off frequencies at which the amplifier's Gain starts rolling down from its Midband value or maximum value, the frequency span between these two cut-off frequencies called the Passband or BandWidth (BW) of the amplifier, the relationship between frequency respond and Gain at constant $\mathrm{Rg}$ varying input voltage for each amplifiers' stage (5) was determined graphically. The result shows that multi stage amplifiers are best suited for the applications which require a high input resistance, low output resistance and large gains.

\section{REFERENCES}

1. Godse, A. P., \& Bakshi, U. A. (2008). Electronic Circuits - I: Technical Publications.

2. Mehta, R. (2008). Objective Electrical Technology: S. Chand Limited.

3. Bhargava, N. N., \& Kulshreshtha,(1983). Basic Electronics And Linear Circuits: Tata Mcgraw-Hill.

4. Ganguly, P. K. (2015). Principles Of Electronics: Prentice Hall India Pvt., Limited.

5. Arora, C. L. (2013). S.Chand's Success Guide R/C B.Sc Physics Vol -3: S. Chand Limited.

6. Navas, K. A. (2015). Electronics Lab Manual Volume I, Fifth Edition: Prentice Hall India Pvt., Limited.

7. Bakshi, U. A., \& Godse, A. P. (2009). Electronic Circuits: Technical Publications.

8. Graham, B., \& Mcgowan, K. (2011). 101 Spy Gadgets For The Evil Genius 2/E: Mcgraw-Hill Education.

9. Godse, A. P. (2010). Electronic Devices And Circuits I: Technical Publications.

10. Dokić, B. L., \& Blanuša, B. (2014). Power Electronics: Converters And Regulators: Springer International Publishing.

11. Johnson, A. (2015). Ap® Physics 1 Crash Course Book + Online: Research \& Education Association.

12. Avison, J. (2014). The World Of Physics: Nelson.

13. Kularatna, N. (2017). Electronic Circuit Design: From Concept To Implementation: Crc Press.

14. Járos, G. G., \& Meyer, B. J. (2013). Elementary Medical Biophysics: Elsevier Science. 